\title{
Predicting farmers' adoption of some agricultural innovations in a village in Assiut governorate, Egypt
}

\author{
Diab A. M. ${ }^{\mathrm{a}^{*}}$, Abdel-Ghany M. M. M. ${ }^{\mathrm{b}}$ \\ ${ }^{a}$ Department of Rural Sociology \& Agricultural Extension, Faculty of Agriculture, New Valley University, Egypt \\ ${ }^{b}$ Department of Rural Sociology \& Agricultural Extension, Faculty of Agriculture, Assiut University, Assiut, Egypt
}

\begin{abstract}
The current research aimed in general to predict the future of farmers' adoption of two agricultural innovation (silage and compost manufacture) through: 1) assessing the studied innovations based on Adoption and Diffusion Outcome Prediction Tool (ADOPT) from farmer views, 2) Predicting the adoption of studied innovation (Predicted peak level of adoption and Predicted years to peak adoption), and 3) determining the factors affecting the adoption. Data were collected during Feb. 2020 using the Focus Group Discussion with members of the board of directors of Local and Agricultural Community Development Association (11 members) in Al-Duwair village of Sidfa District, Assiut governorate, Egypt. Results of the study found that both the studied innovation (Silage and Compost) will achieve the peak level of adoption (98\%) during 4, 10 years, respectively. The study also found that the factors associated with the design and characteristics of the innovation were at the forefront of the factors affecting the adoption of the studied innovations, followed by factors related to extension efforts. In light of the results reached, the study recommended the use of ADOPT in predicting all agricultural innovations before starting the diffusion, because the tool was designed for this purpose, and the results of the study can be used to accelerate access to the peak level of adoption of the studied innovations by affecting the internal components of the ADOPT.
\end{abstract}

Keywords: adoption, diffusion, adoption prediction, adopt, silage, compost.

\footnotetext{
* Corresponding author: Diab A. M.,
} 


\section{التنبؤ بتبني الزر اع لبعض المستحدثات الزر اعية بإحدى قرى محافظة أسيوط بجمهورية مصر العربية \\ أحمد محمد دياب' ، محمد محمد محمد عبد الغني '

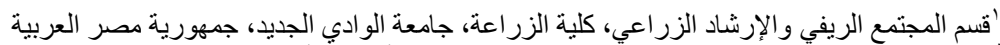

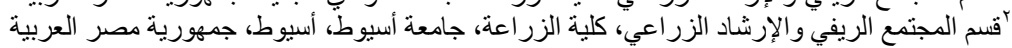

المستخلص

استهدف البحث بصفة عامة التتبؤ بتبني المزارعين لكل من مستحثى تصنيع السيلاج وتصنيع الكومبوست، وذلك من خلال: ( ) تقييم

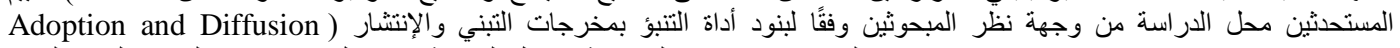

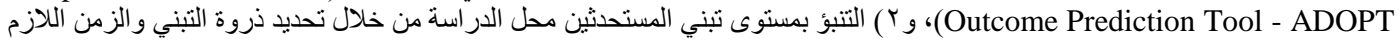

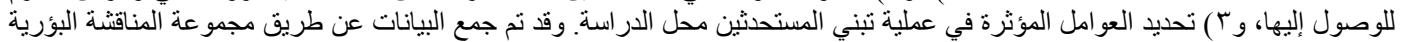
Focus Group Discussion

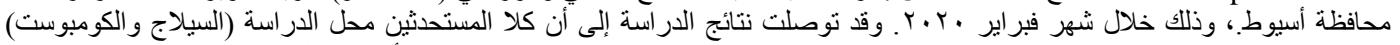

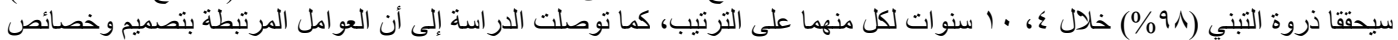

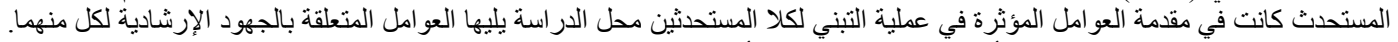

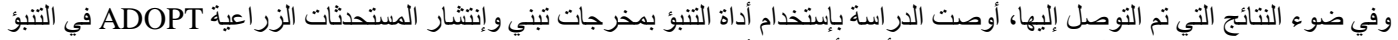

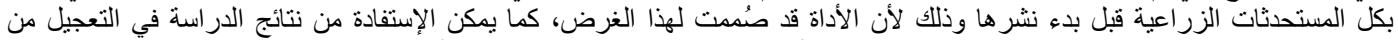
الوصول إلى ذروة التبني للمستحدثات المدروسة من خلاتل التأثنير على العناصر الداخلية المكونة للأداة. 


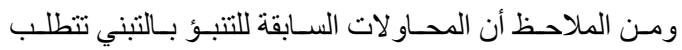

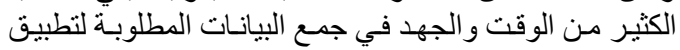

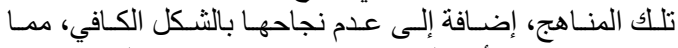

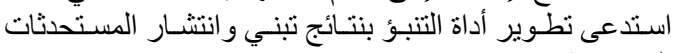

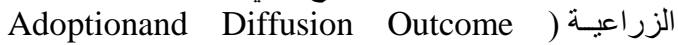
Kuehne بواسطة (Prediction Tool (ADOPT)

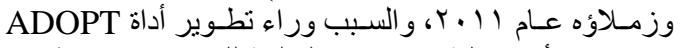

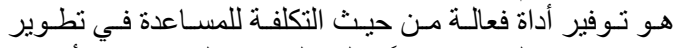

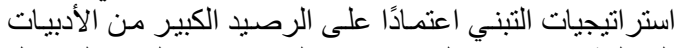

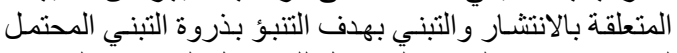

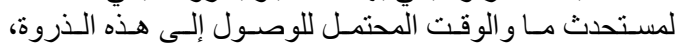

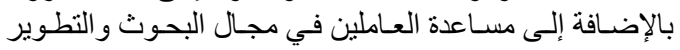

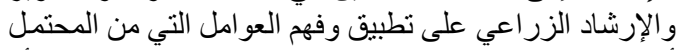

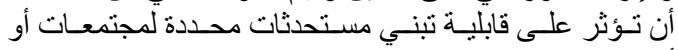

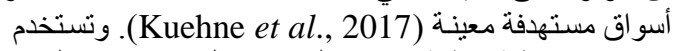

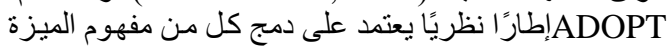

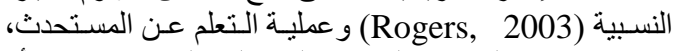

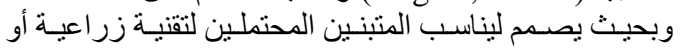

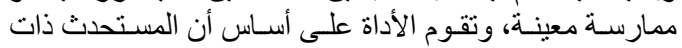

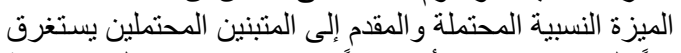

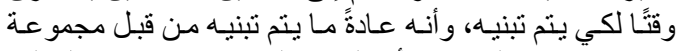

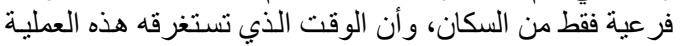

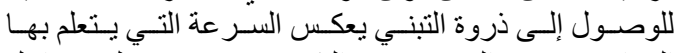

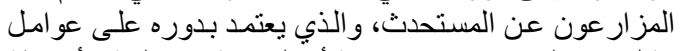

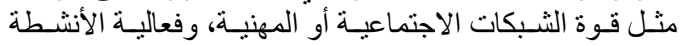

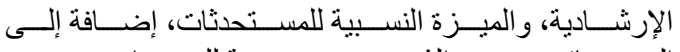

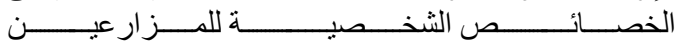

Rogers, 2003; Llewellyn and Brown, 2020; ) (Pannell et al., 2006, فالمستحدث يشير إلى أب فكرة أو أو أنها.

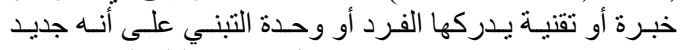

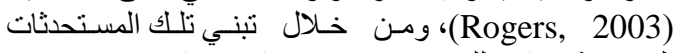

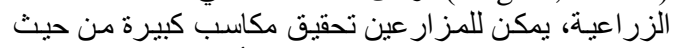

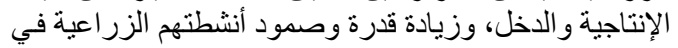

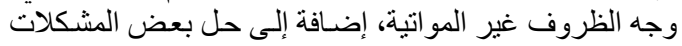

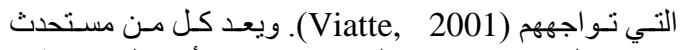

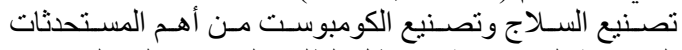

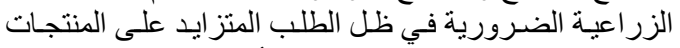

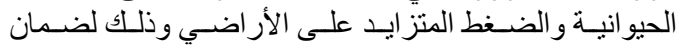

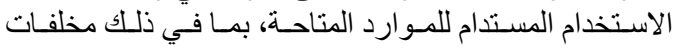

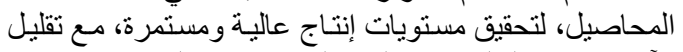

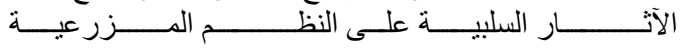
(Rota and Sperandini, 2010)

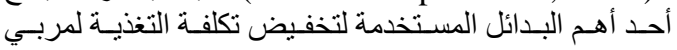

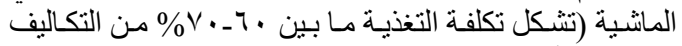

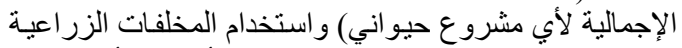

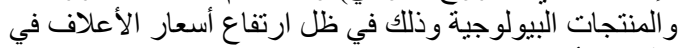

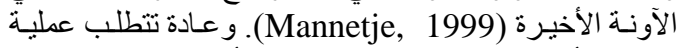
تحويل الأعلاف الخضر اء إلى سيلاج لأماكن محصورة عن
مقدمة - مقة

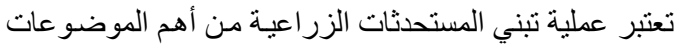

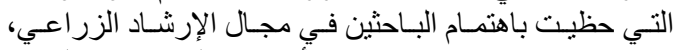

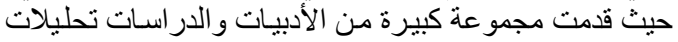

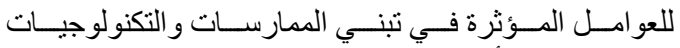

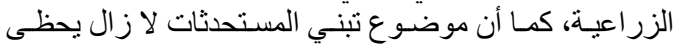

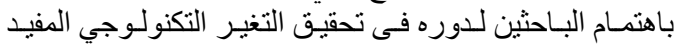

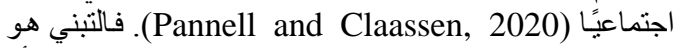

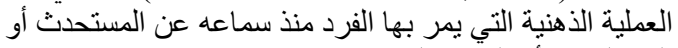

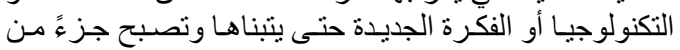

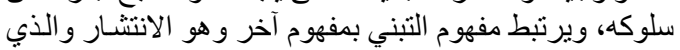

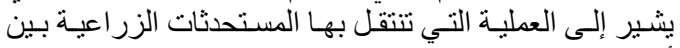

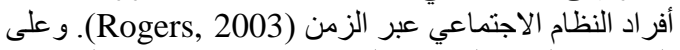

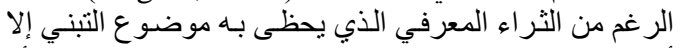

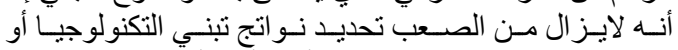

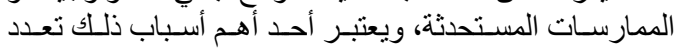

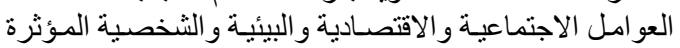

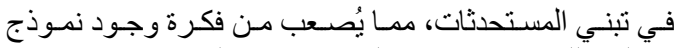

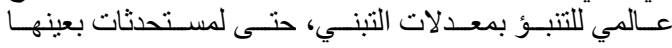
Baumgart-Getzet et al., 2012; Knowler and ) Bradshaw, 2007; Munguia and Llewellyn,

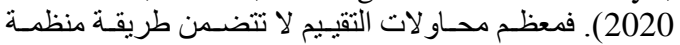

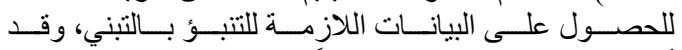

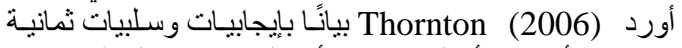

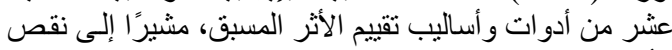

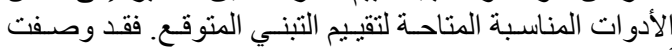

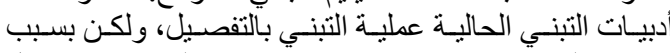

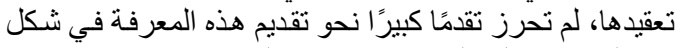

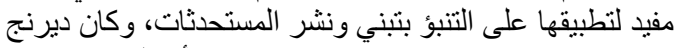

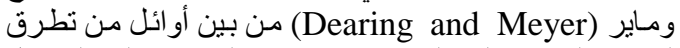

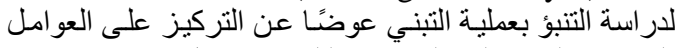

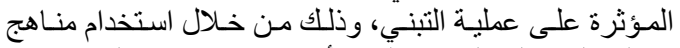

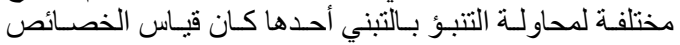

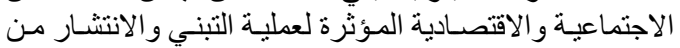

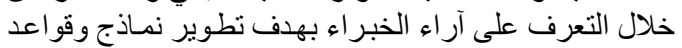

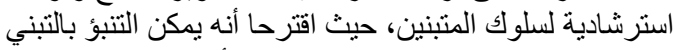

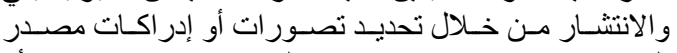

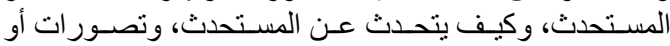

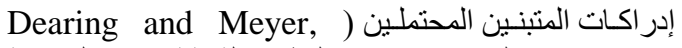

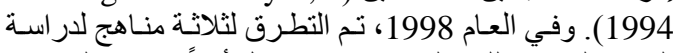

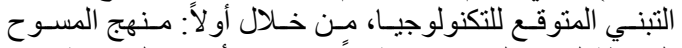

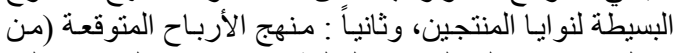

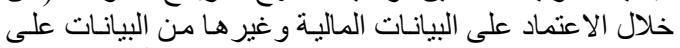

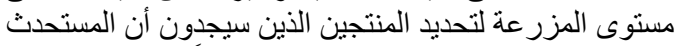

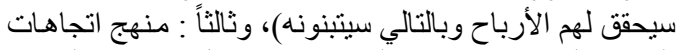

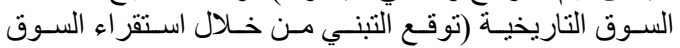
المستقلية لاستخدام المستحدث) (Caswell et al., 1998). 


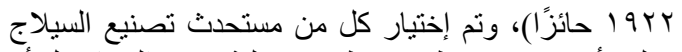

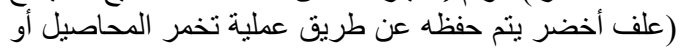

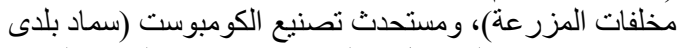

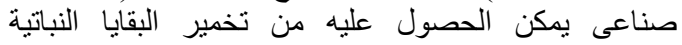

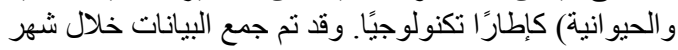

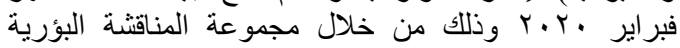
مع أعضاء مجلس إدارة (Focus Group Discussion)

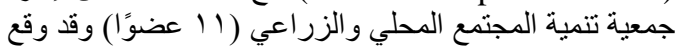

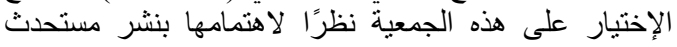

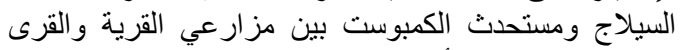

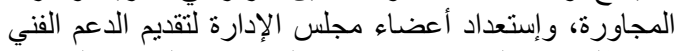

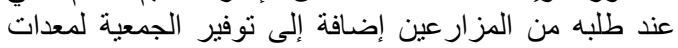

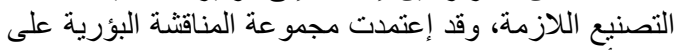

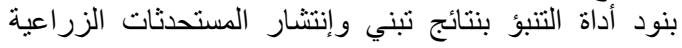
Adoption and Diffusion Outcome Prediction ) (Tool (ADOPT) ) حول كل من المستحدثين محل الدراسة. وتستتد $\quad$ ADOPT الأداة (ADT) (https://adopt.csiro.au

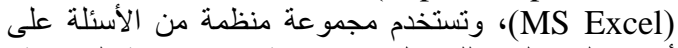

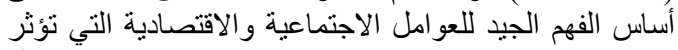

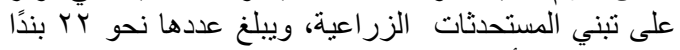

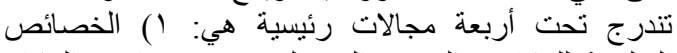

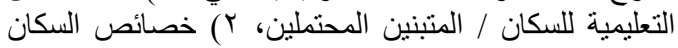

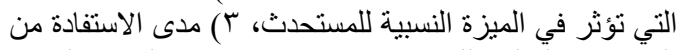

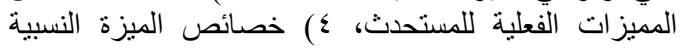

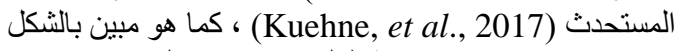

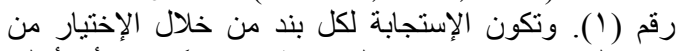

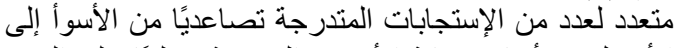

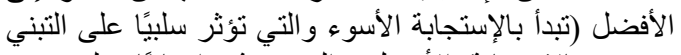

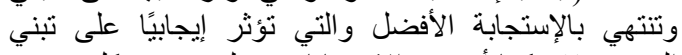

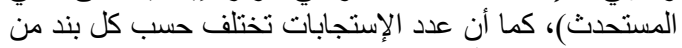

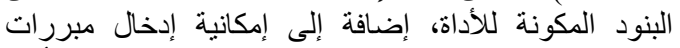

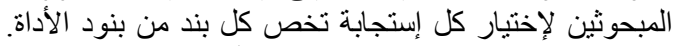

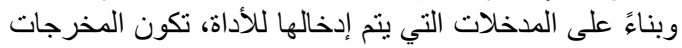

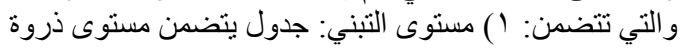

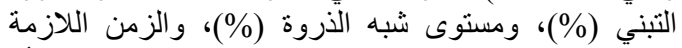

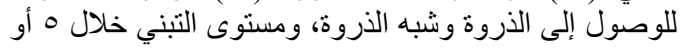

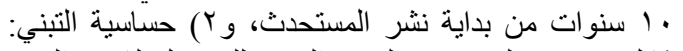

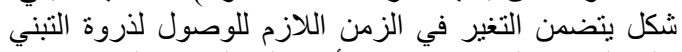

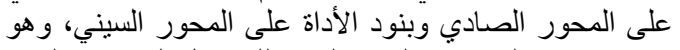

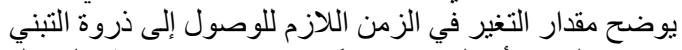

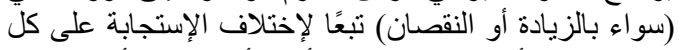

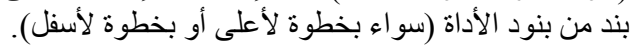

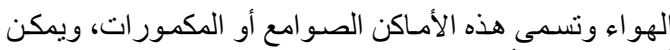

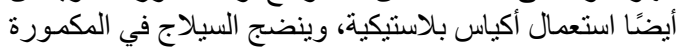

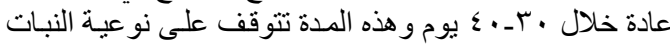

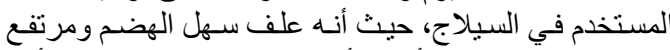

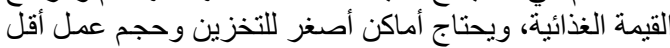

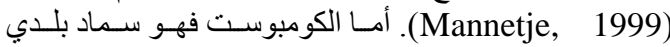

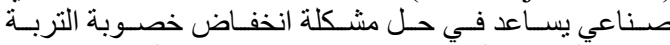
وارتفاع أسعار الأسمدة الكيماوية إضـافة إلى التأثيرات النيات السليية

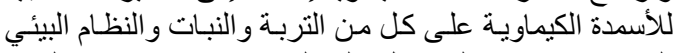

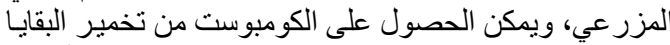

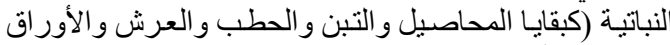

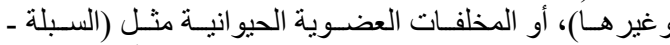

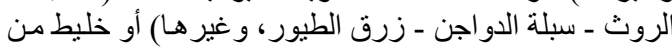

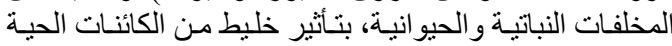

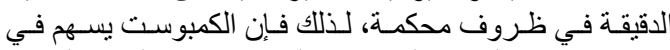

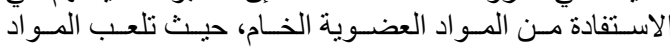

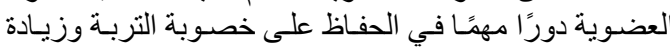

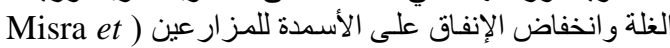

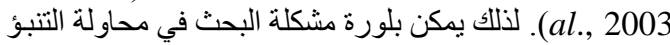

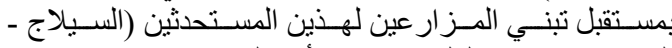

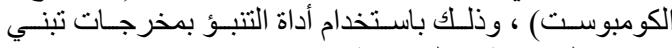
و إنتشار المستحدثات الزراعية (ADOPT).

أهداف البحث بناءً على العرض السابق، يهدف البحث الحالي إلى:

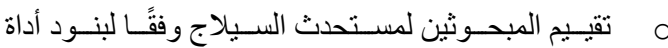
ADOPT

O تقبيم المبحــوثين لمســــدث الكمبوسـت وفقًا لبنـود أداة ADOPT

o التنبؤ بمستوى تبني مستحدث السيلاج من خلال تحديد

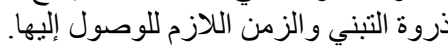

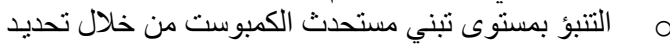

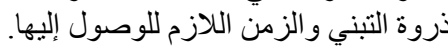
م تحديد العوأمل المؤثرة في عملية تبني المستحدثين محل

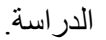

\section{الطريقة البحثية ومصادر جمع البيانات}

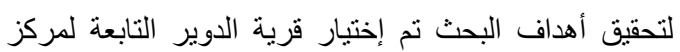

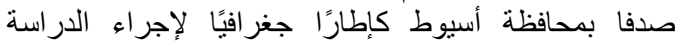

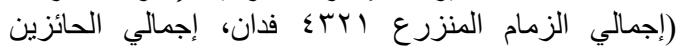




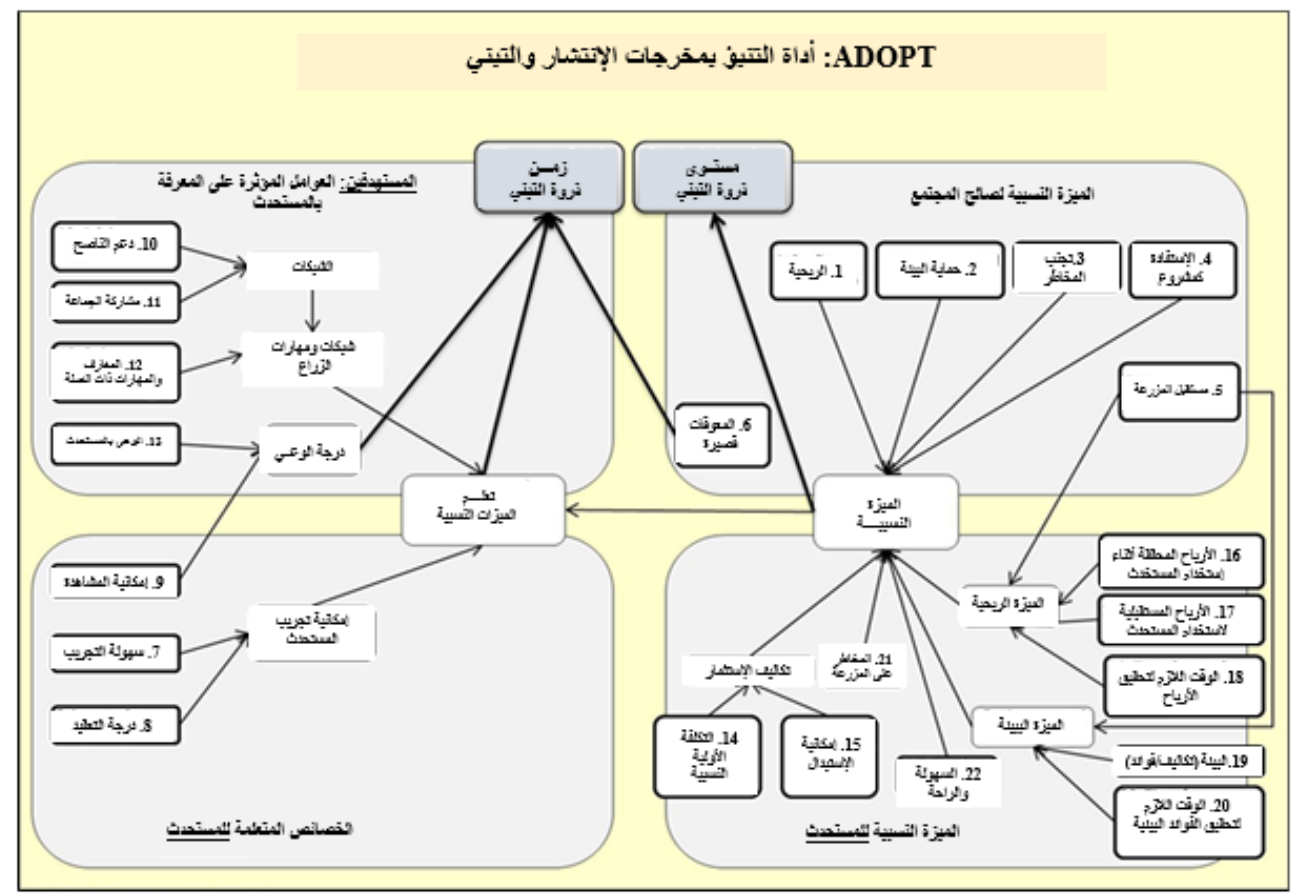

شكل ( (1): أداة التنبؤ بمخرجات إنتشار وتبني المستحدثات. المصدر: (Kuehne et al., 2017).

ب. تقييم المبحوثين لمستحدث تصنيع الكومبوست وفقًا لبنود

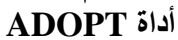

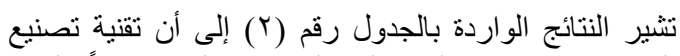

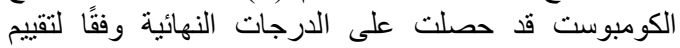

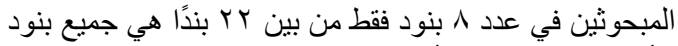

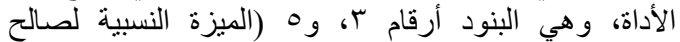

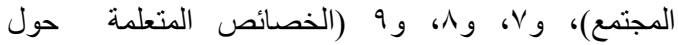

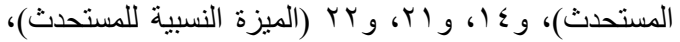

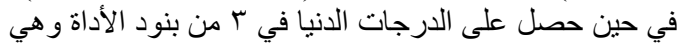

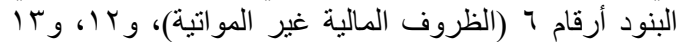

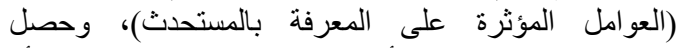

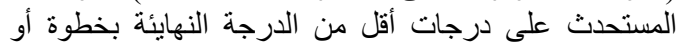

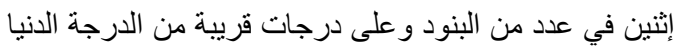

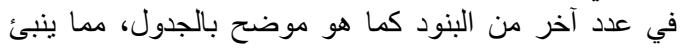

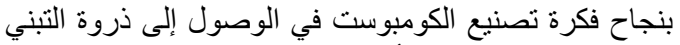

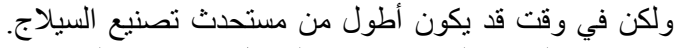

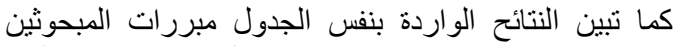

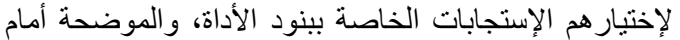

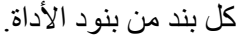

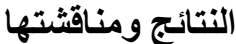

أولا: تقييم المبحوثين للمستحدثات محل الدراسة وفقًا لبنود أداة التتبؤ بمخرجات التبني والإنتشار لقين

أ. تقييم المبحوثين لمستحدث تصنيع السيلاج وفقًا لبنود أداة

ADOPT

تثبير النتائج الواردة بالجدول رقم (1) إلى أن تقنية تصنيع

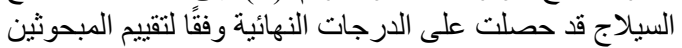

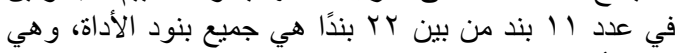

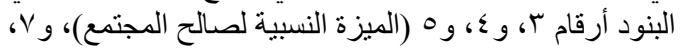

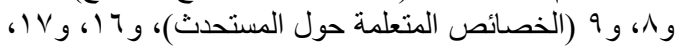

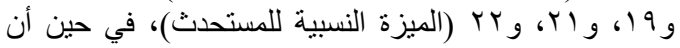

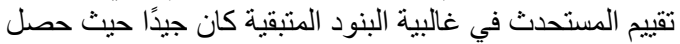

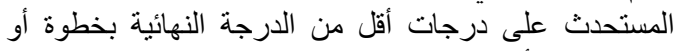

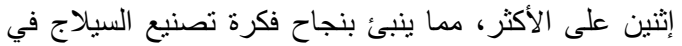

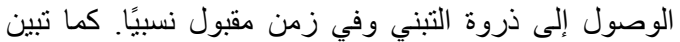

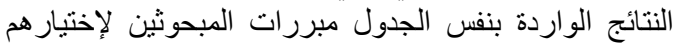
الإستجابات الخاصة بينود الأداة، و الموضحة أمام كل بند. لإند 
Diab A and Abdel-Ghany / Archives of Agriculture Sciences Journal 3(2) 145-155, 2020.

جدول ( (): نتائج مجمو عة المناقثة البؤرية المتعلقة بإستجابات ومبررات المبحوثين على بنود أداة ADOPT بخصوص مستحدث تصنيع السبلاج.

\begin{tabular}{|c|c|c|c|}
\hline مبررات الاستجابات & الاستجابات & الاستجابات & 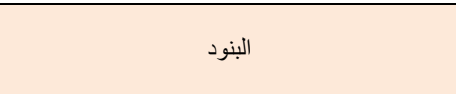 \\
\hline تقليل إستخدام العلائق الجاهزة الأخرى بلى الإضافة إلى & أز الزاع البية من & 0 & 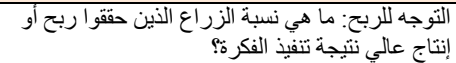 \\
\hline 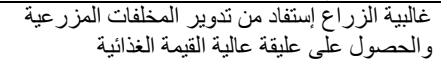 & أز الزاعاعية من & 。 & 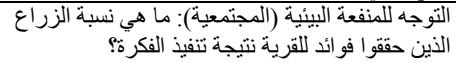 \\
\hline و لا أحد يحتب التعرض لأي فخاطميع يرضرة النجاح في زر اعتة & 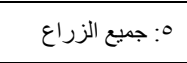 & 0 & 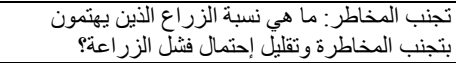 \\
\hline 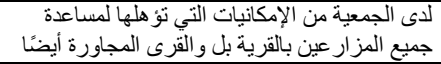 & 0 ميع الزراع & $\circ$ & 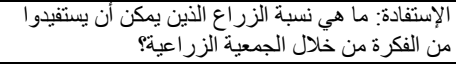 \\
\hline 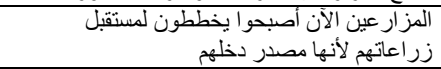 & 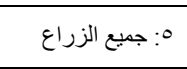 & 0 & لمستقبل زر المزعة عة: ما هي نسبة الزر اع الذين يخططون \\
\hline 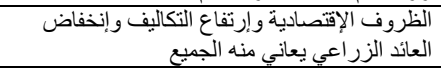 & 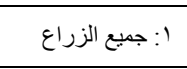 & 0 & 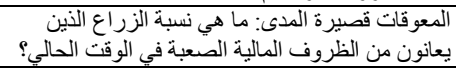 \\
\hline 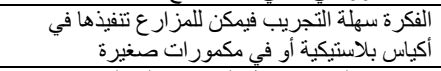 & تجربة الفكرة جندًا & 0 & تطبيقها ألتريب: ما مدى سهولة تجريب الفكرة قبل \\
\hline 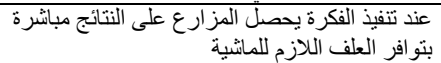 & 0 : سهلة جذًا & 0 & بسهوجة التعقيد: هل بمكن معرفة نتائج استخدام الفكرة \\
\hline 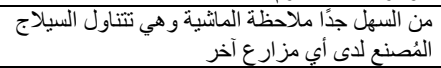 & 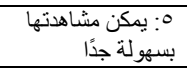 & 0 & 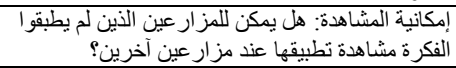 \\
\hline 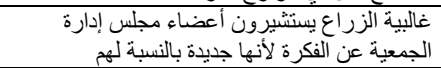 & 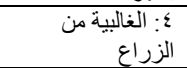 & 0 & 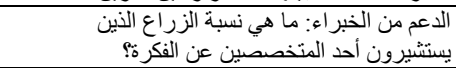 \\
\hline 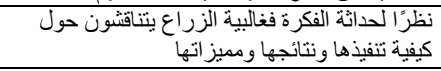 & أز أزراع البية من & 0 & 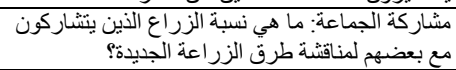 \\
\hline 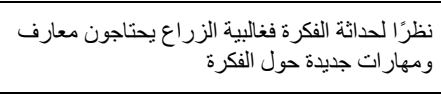 & الز إ: الغالبية من & 0 & 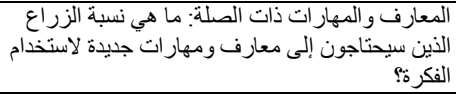 \\
\hline 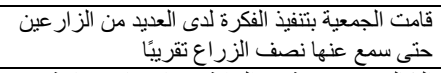 & 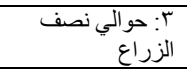 & 0 & 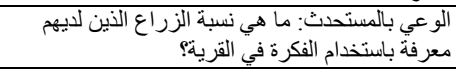 \\
\hline 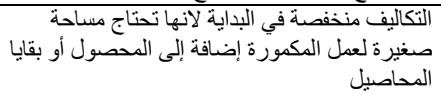 & البداية & 0 & التطلبها استخدام الفكرة: ؟ الألية النسبية مقار التكاليف التي \\
\hline في عمل الكومبوست الفكرة بمكن ردم الحفرة أو إستخدامها & ع يمكن بسهولة & 0 & 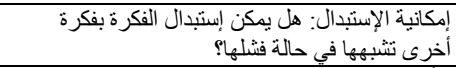 \\
\hline الأرباح تصل إلى . . †\% من التكلفة الفعلية & ^: أرباح كبيرة جذًا & $\wedge$ & 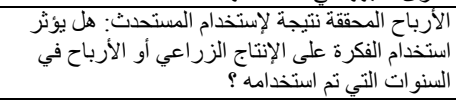 \\
\hline الأرباح تصل إلى ·. . \% من التكلفة الفعلية & ^: أرباح كبيرة جذًا & $\wedge$ & 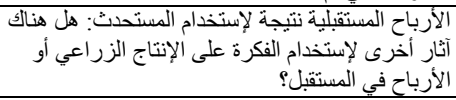 \\
\hline بظدهر تأثير الذفرة وسبلجنها السنة الأولى من تنفيذها، & 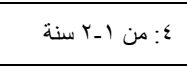 & 1 & 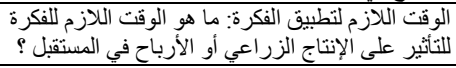 \\
\hline 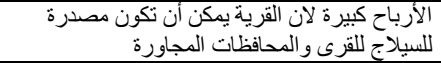 & ^^ أرباح كبيرة جدًا & $\wedge$ & 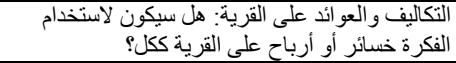 \\
\hline تحقيق الأرباح يظهر خلال أول سنة من التطبيق & 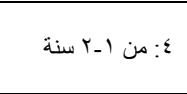 & 0 & 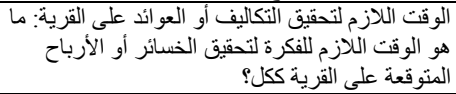 \\
\hline 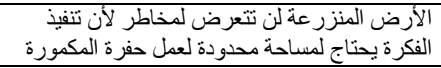 & في المخاطر كبير جدًا & $\wedge$ & على تعرض على الأرض المزف: هل سيؤثر استخدام الفكرة \\
\hline 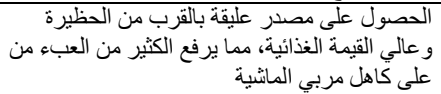 & في السهولة والراحة جيادة & $\wedge$ & الزراعة في السنوة : هل أثر استخدام الفكرة على سهولة \\
\hline
\end{tabular}


جدول (r)): نتائج مجمو عة المناقثة البؤرية المتعلقة بإستجابات ومبررات المبحوثين لبنود أداة ADOPT بخصوص مستحدث تصنيع الكومبوست.

\begin{tabular}{|c|c|c|c|}
\hline مبررات الاستجابات & الاستجابات & الاستجابات & 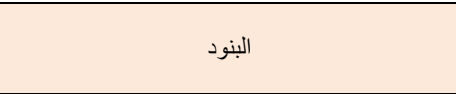 \\
\hline 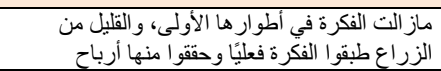 & 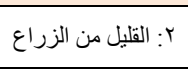 & 0 & إنتاج عالي نتنيجة تنفيذ الفكرة؛ الزراع الذين حققو اربح أو \\
\hline 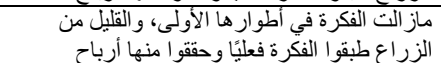 & 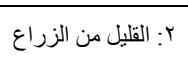 & 。 & 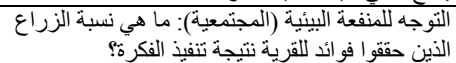 \\
\hline و لا أحد التعرضب الفثل فالجميع بريد النجاح في زر اعتة & 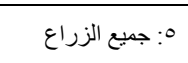 & 。 & 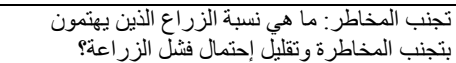 \\
\hline 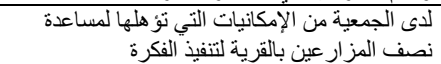 & 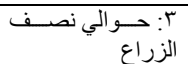 & 。 & 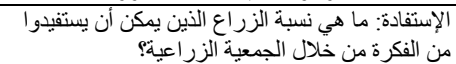 \\
\hline 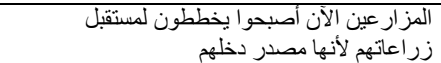 & 0: جميع الزراع & ○ & لمستقبل زالمزاعة أرضة: ما هي نسبة الزر اع الذين يخططون \\
\hline الظائد الزر الإقي بعانية ونه الجميع التكاليف و إنخفاض & 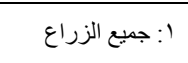 & 。 & يعانون من الظبروف المالية الصعبة في المبة الزرقت الحالي؟ \\
\hline أكياس بلاستيكية أو في في فكمور للمزات صغيرة تنفيذها في & تجربة الفكرة & 0 & تطبيقها إنجريب: ما مدى سهولة تجريب الفكرة قبل \\
\hline 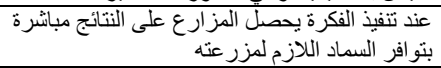 & 0: سهلة جدًا & 0 & بسهولة ب التعقيد: هل يمكن معرفة نتائج استخدام الفكرة \\
\hline مز ارع آخرل جدًا ملاحظة الكومبوست المُصنع لدى أي & 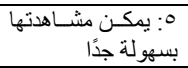 & o & إلفكرة مشاهدة المشادة: هل يمكا عند للمز ارعين آخرين الذين لم يطبقو ا \\
\hline 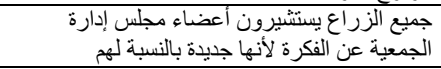 & • : جميع الزراع & o & 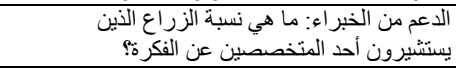 \\
\hline 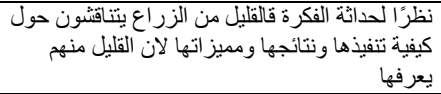 & ب آ: القليل من الزراع & 0 & 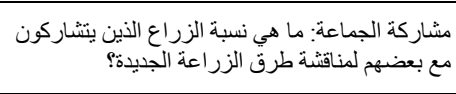 \\
\hline ومهار ات جديدة حول الفكرة فيعرة الزر اع يحتاجون معارف & 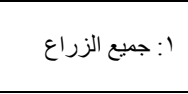 & 。 & 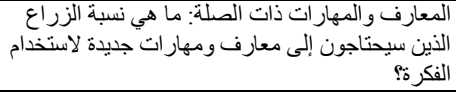 \\
\hline لكبس لدى المز ار عين بالقرية أبة معرفة بطريقتّصنيع & إ: لا أحد & 0 & 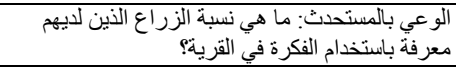 \\
\hline 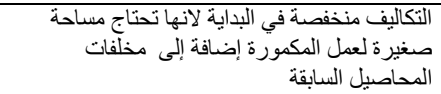 & في البداية الحتاج تكاليف & o & يتطلبها استخدام الفكرة ؟ الأولية ما هو مقدار التكاليف التي \\
\hline في عمل الكو مبوست مثلغً & ؟ : يمكن بسهولة & 。 & 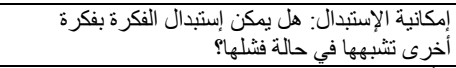 \\
\hline الأرباح جيدة وتتمثل في توفير أسعار الأسمدة & 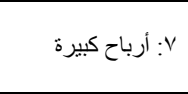 & $\wedge$ & 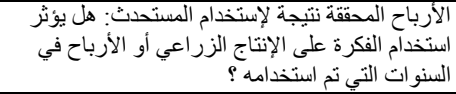 \\
\hline الكيماوية مرتفعة الثمن في توفير أسعار الأسمدة & 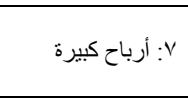 & $\wedge$ & 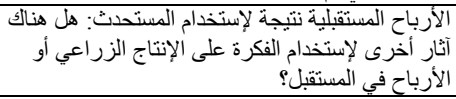 \\
\hline يظهر تأثير الفكرة خلال السنة الأولى من تتفيذها & 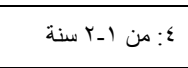 & 1 & 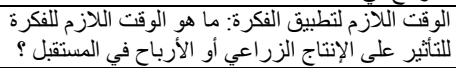 \\
\hline 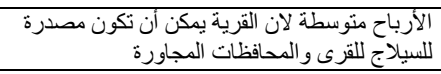 & 7 آ أرباح متوسطة & $\wedge$ & الفكرة خسائر أو أدرباح على القية: هل سيكون كلى لاستخدام \\
\hline تحقيق الأرباح يظهر خلال أول سنة من النطبيق & 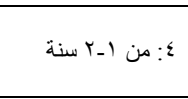 & 。 & 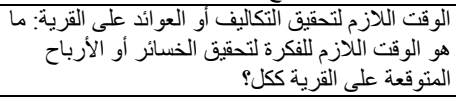 \\
\hline 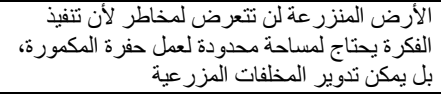 & في المخاطر كبير جذًا & $\wedge$ & المخلىر عترض الأرض المزر المزرو هل سيؤثر استخدام الفكرة \\
\hline و و الحصيقة آمنه في التخلص من بيد في مكان المزرعيل & في السيولة وبيرة جداًا & $\wedge$ & 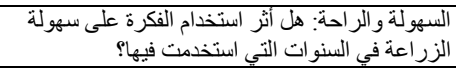 \\
\hline
\end{tabular}

المصدر: حسبت من: بيانات الجدول رقم (1). 


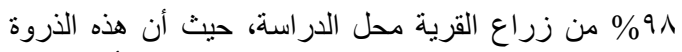

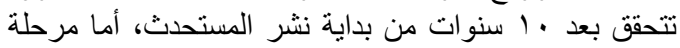

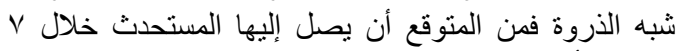

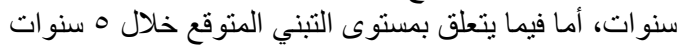

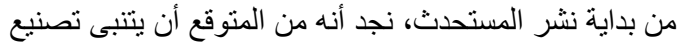

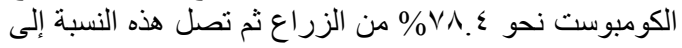

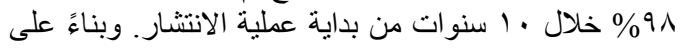

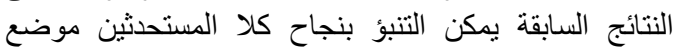

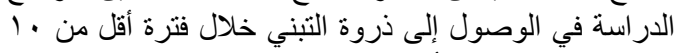

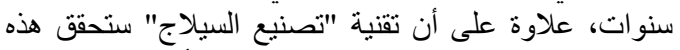

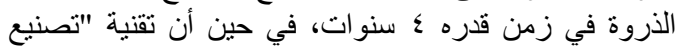

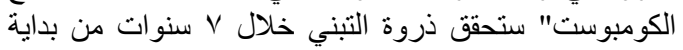

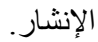

تبين النتائج الواردة بالجدول رقم (T) أن ذروة التبني الخاصة

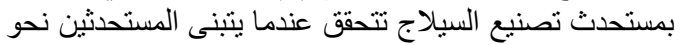

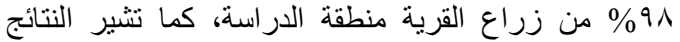

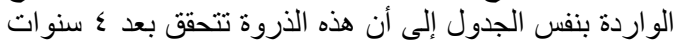

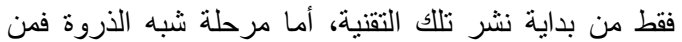

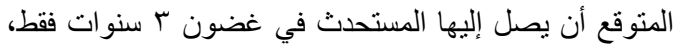
أما فيما يتعلق بمستوى التبني المنتوقع خلال ه سنو المن ات من بداية

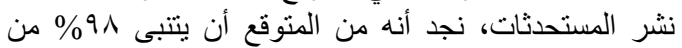

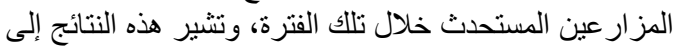
النجاح المنوقع حدوثة لمسنوى تبني مستحدث تصنئ تصنيع السيلاج

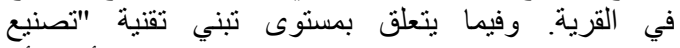

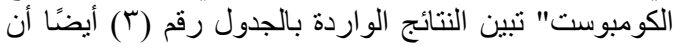

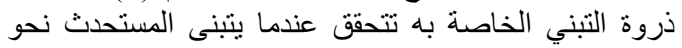

جدول (r): مستوى ذروة وزمن التبني المتوقع للمستحدثين محل الدراسة.

\begin{tabular}{|c|c|c|}
\hline تصنيع الكو مبوست (\%) & تصنيع السيلاج (\%) & مستوى ذروة وزمن التبني المتوقع \\
\hline 91 & 91 & مستوى ذروة التبني المتوقع \\
\hline 1. & $\varepsilon$ & السنو ات المتوقعة لبلو غ ذروة التبني \\
\hline V & $r$ & سنو ات متوقعة لبلوغ شبه الذروة \\
\hline$\vee \wedge . \varepsilon$ & $9 \wedge$ & مستوى التبني المتوقع خلال ه سنوات من البداية \\
\hline 91 & $9 \wedge$ & مستوى التبني المتوقع خلال • سنوات من البداية \\
\hline
\end{tabular}

المصدر: نتائج الدراسة بإستخدام أداة ADOPT.

لأسفل (أي إختبار المبحوثين للإستجابة السابقة مباشرة

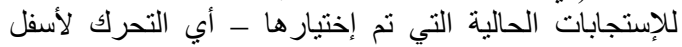

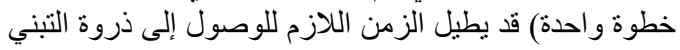

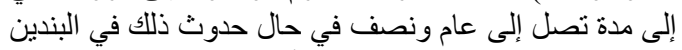

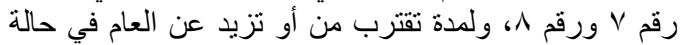

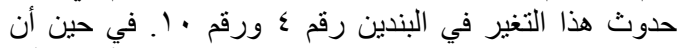

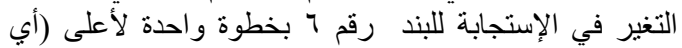

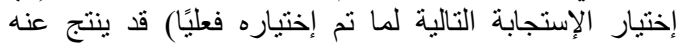

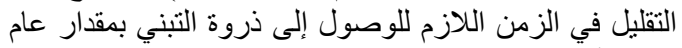

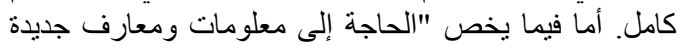

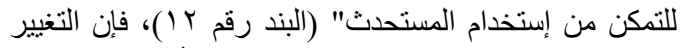

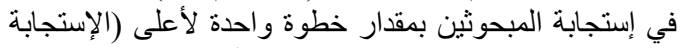

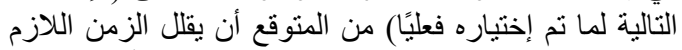

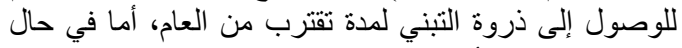

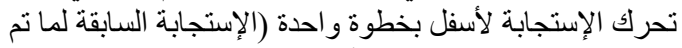

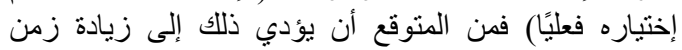

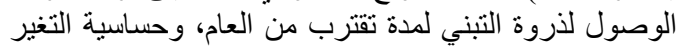

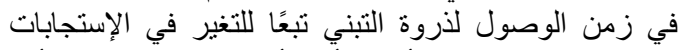

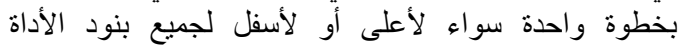

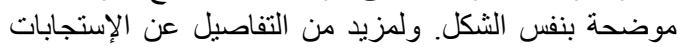

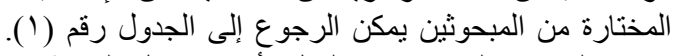

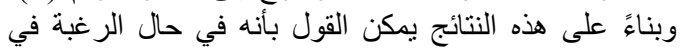

ثالثا: العوامل المؤثرة في مستوى تبني المستحثين محل الدراسة

\section{أ. العوامل المؤثرة في مستوى تبني مستحدث تصنيع السيلاج}

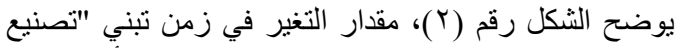

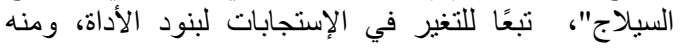

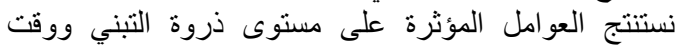

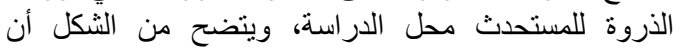

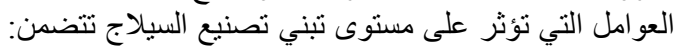

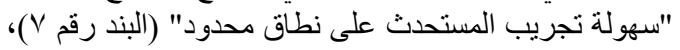

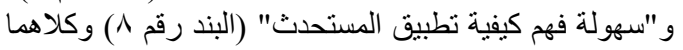

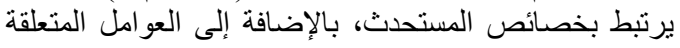

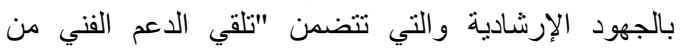

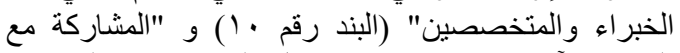

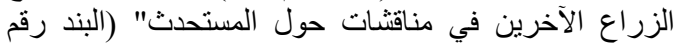

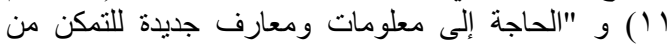

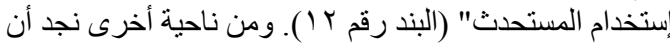

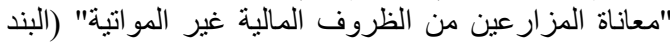

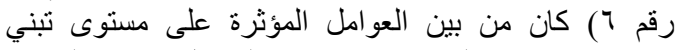

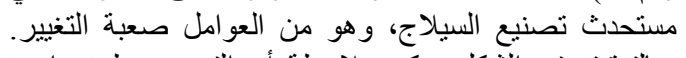
وبالتدقيق في الثكل بمكن ملاحظة أن التغير بخطوة واحدة الثنا 


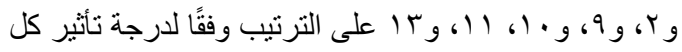

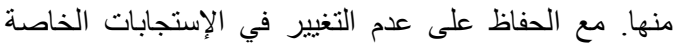

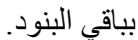

تسريع الوصول إلى ذروة تبني مستحدث تصنيع السيلاج

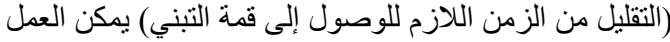

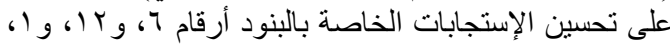

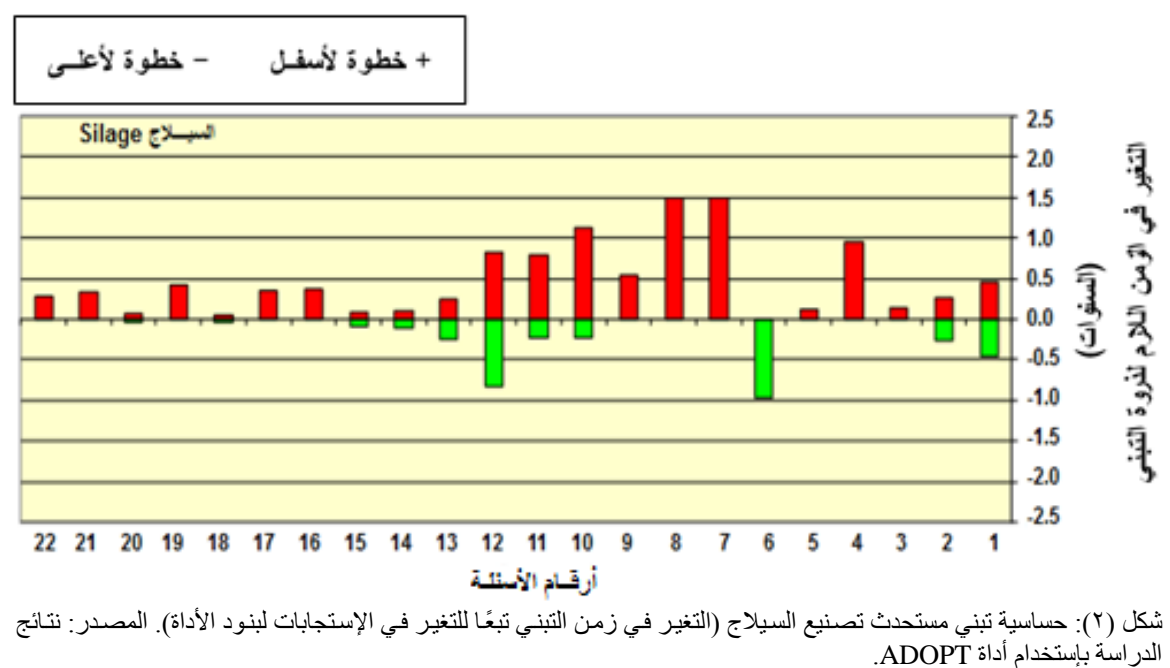

للوصول إلى ذروة التبني لمدة تصل إلى عام ونصف في في حال

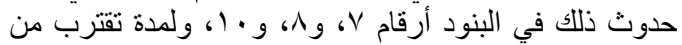

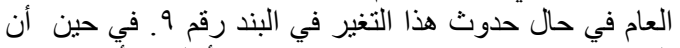

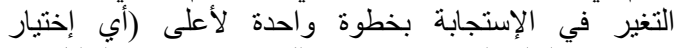

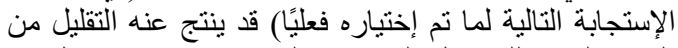

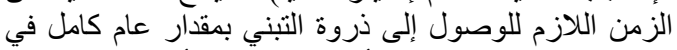

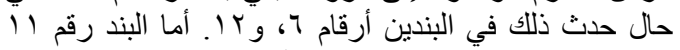

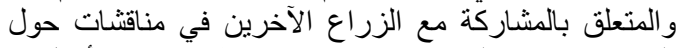

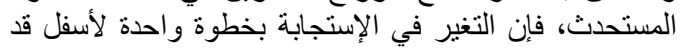

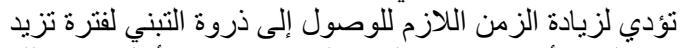

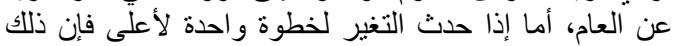

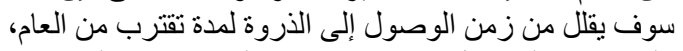

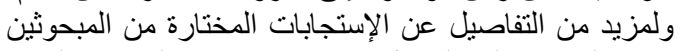

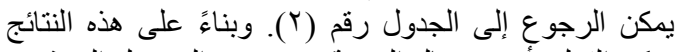

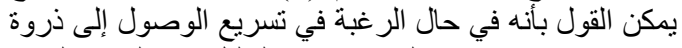

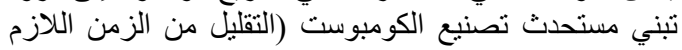

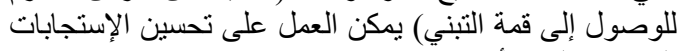

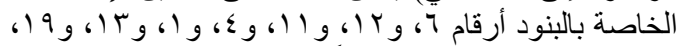

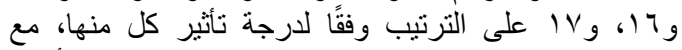
الحفاظ على عدم التغيير في الإستجابات الخاصة بالئل ولينود أرقام

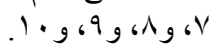

\section{ب. العوامل المؤثرة في مستوى تبني مستحدث تصنيع

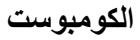

تبين النتائج الواردة بالثكل رقم (با)، مقدار التغير في زمن

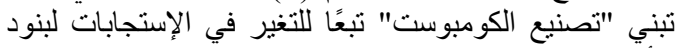

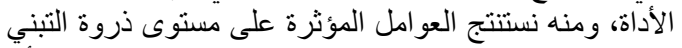

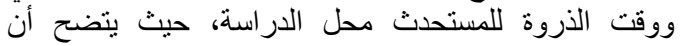

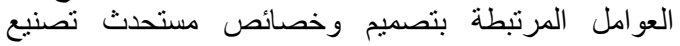
الكومبوست كانت في مقدمة العو امل المؤثرة في عملية التبني لنئي

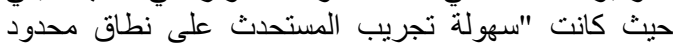

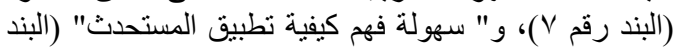

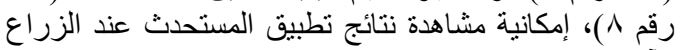

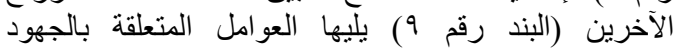
الإرشادية والتي تتضمن "تلقي الدعم الفني من النئ الخبراء

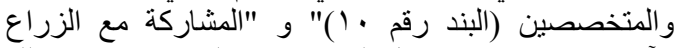

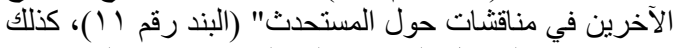

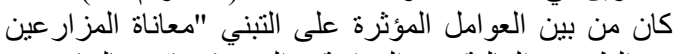

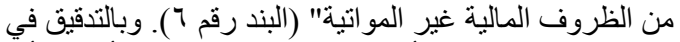

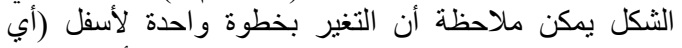

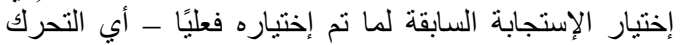

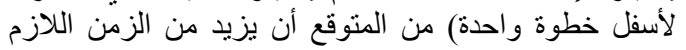




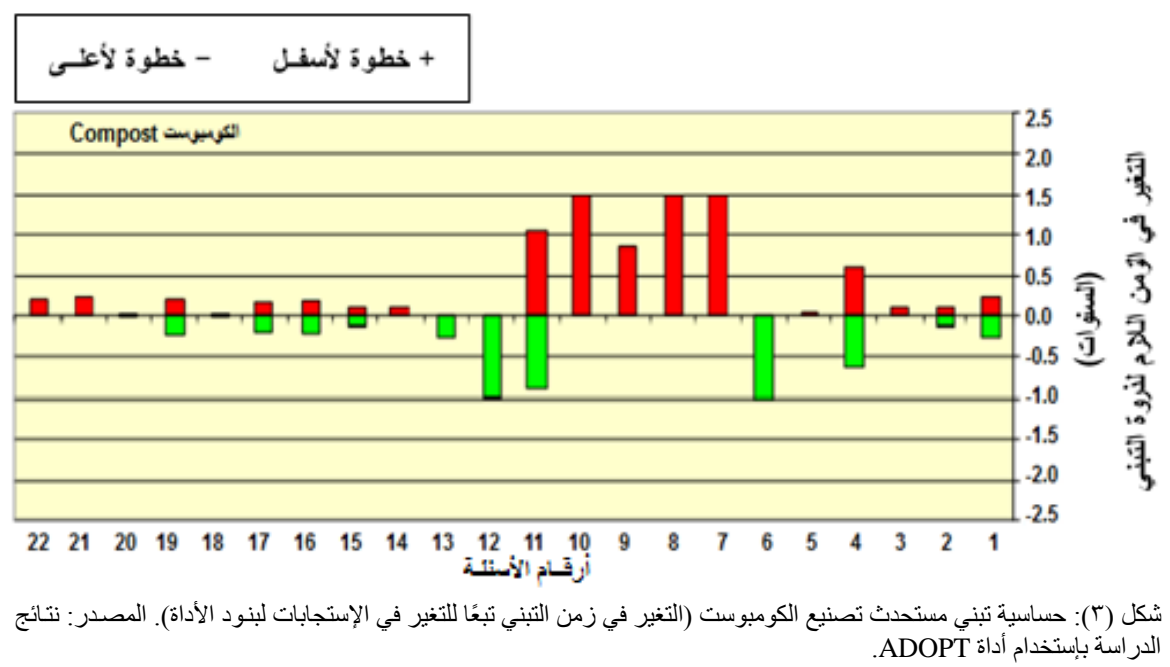

في مقدمة العوامل المؤثرة في عملية تبني كلا المستحدثين

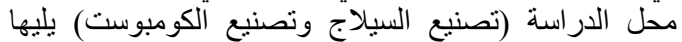

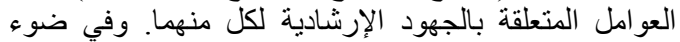

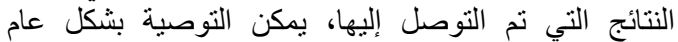

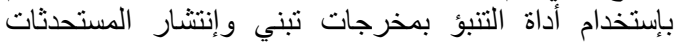

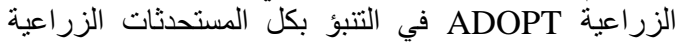

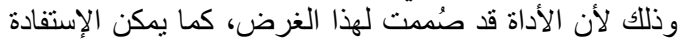

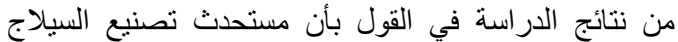

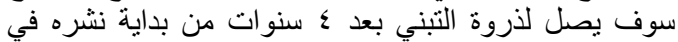

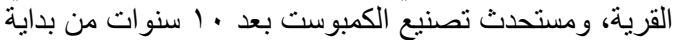

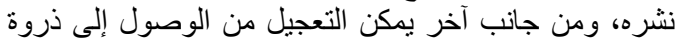

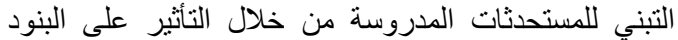

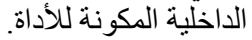

Baumgart-Getz, A., Prokopy, L. S. and Floress, K. (2012), "Why Farmers Adopt Best Management Practice in the United States: A Meta-Analysis of the Adoption Literature", Journal of Environmental Management, Vol. 96, pp. 17-25.

Caswell, M. F., Fuglie, K. O. and Klotz, C. A. (1994), Agricultural Biotechnology: An Economic Perspective, Agricultural Economics Reports 262025, Economic

\section{الخلاصة و التوصيات}

توصلت نتائج الدراسة إلى أن مستوى ذروة التبني للمستحدثين

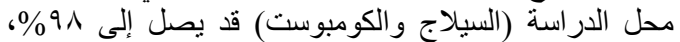

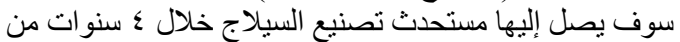

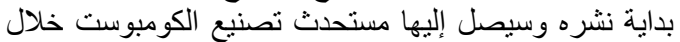

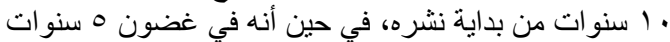
من بداية أنشطة نشر كلا المستحدثين، سيكون تصنيع النيع السيلاج

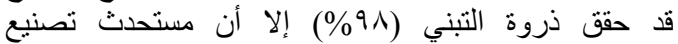

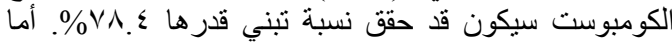

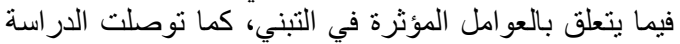

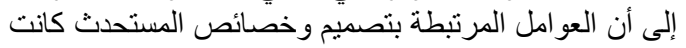

\section{قائمة المراجع}

Research Service, Department of Agriculture, United States.

Dearing, J. and Meyer, G. (1994), "An exploratory tool for predicting adoption decisions", Science Communication, Vol. 16 No. 1, pp. 43-57.

Knowler, D. and Bradshaw, B. (2007), "Farmers' Adoption of Conservation Agriculture: A Review and Synthesis of 
Recent Research", Journal of Food Policy, Vol. 32, pp. 25-48.

Kuehne G., Llewellyn, R., Pannell, D., Wilkinson, R., Dolling, P., Ouzman, J. and Ewing, M. (2017), "Predicting farmer uptake of new agricultural practices: A tool for research, extension and policy", Agricultural Systems, Vol. 156, pp.115125.

Llewellyn, R. S. and Brown, B. (2020), "Predicting Adoption of Innovations by Farmers: What is Different in Smallholder Agriculture?", Applied Economic Perspectives and Policy, Vol. 42 No. 1, pp. 100-112.

Mannetje, L. (1999), Silage making in the tropics with particular emphasis on smallholders, Proceedings of the FAO Electronic Conference on Tropical Silage, Plant Production and Protection Paper, Food and Agriculture Organization of the United Nations, Italy.

Misra, R. F., Roy, R. N. and Hiraoka, H. (2003), On-Farm Composting Methods, Land and water discussion paper, Food and Agriculture Organization, Rome, Italy.

Munguia, O. M. O. and Llewellyn, R. (2020), "The adopters versus the technology: Which matters more when predicting or explaining adoption", Applied Economic Perspectives and Policy, Vol. 42 No. 1, pp. 80-91.
Pannell, D. J. and Claassen, R. (2020), "The Roles of Adoption and Behavior Change in Agricultural Policy", Applied Economic Perspectives and Policy, Vol. 42 No. 1, pp. 31-41.

Pannell, D. J., Marshall, G. R., Barr, N., Curtis, A., Vanclay, F. and Wilkinson R. (2006), "Understanding and Promoting Adoption of Conservation Technologies by Rural Landholders", Australian Journal of Experimental Agriculture, Vol. 46, pp. 1407-1424.

Rogers, E. (2003), Diffusion of Innovations, New York, Free Press, USA.

Rota, A. and Sperandini, S. (2010), Integrated Crop-Livestock Farming Systems, Livestock Thematic Papers Tools for Project Design, International Fund for Agricultural Development (IFAD), Rome. Italy.

Thornton, P. K. (2006), "Ex ante impact assessment and seasonal climate forecasts: Status and Issues", Journal of Climate Research, Vol. 33, pp. 55-65.

Viatte, G. (2001), Adopting technologies for sustainable farming systems: An OECD perspective, Wageningen Workshop Proceeding, Organization for Economic Co-operation and Development (OECD), Paris, France. 